\title{
Development and Validation of Liquid Chromatographic Method for Estimation of Naringin in Nanoformulation
}

\author{
Kranti P. Musmade, M. Trilok, Swapnil J. Dengale, Krishnamurthy Bhat, M. S. Reddy, \\ Prashant B. Musmade, and N. Udupa \\ Department of Pharmaceutical Quality Assurance, Manipal College of Pharmaceutical Sciences, Manipal University, \\ Manipal 576104, India
}

Correspondence should be addressed to N. Udupa; n.udupa@manipal.edu

Received 19 November 2013; Revised 20 December 2013; Accepted 26 December 2013; Published 6 March 2014

Academic Editor: Massoud Amanlou

Copyright (C) 2014 Kranti P. Musmade et al. This is an open access article distributed under the Creative Commons Attribution License, which permits unrestricted use, distribution, and reproduction in any medium, provided the original work is properly cited.

\begin{abstract}
A simple, precise, accurate, rapid, and sensitive reverse phase high performance liquid chromatography (RP-HPLC) method with UV detection has been developed and validated for quantification of naringin (NAR) in novel pharmaceutical formulation. NAR is a polyphenolic flavonoid present in most of the citrus plants having variety of pharmacological activities. Method optimization was carried out by considering the various parameters such as effect of $\mathrm{pH}$ and column. The analyte was separated by employing a $_{18}(250.0 \times 4.6 \mathrm{~mm}, 5 \mu \mathrm{m})$ column at ambient temperature in isocratic conditions using phosphate buffer $\mathrm{pH}$ 3.5: acetonitrile $(75: 25 \% \mathrm{v} / \mathrm{v})$ as mobile phase pumped at a flow rate of $1.0 \mathrm{~mL} / \mathrm{min}$. UV detection was carried out at $282 \mathrm{~nm}$. The developed method was validated according to ICH guidelines Q2(R1). The method was found to be precise and accurate on statistical evaluation with a linearity range of 0.1 to $20.0 \mu \mathrm{g} / \mathrm{mL}$ for NAR. The intra- and interday precision studies showed good reproducibility with coefficients of variation (CV) less than $1.0 \%$. The mean recovery of NAR was found to be $99.33 \pm 0.16 \%$. The proposed method was found to be highly accurate, sensitive, and robust. The proposed liquid chromatographic method was successfully employed for the routine analysis of said compound in developed novel nanopharmaceuticals. The presence of excipients did not show any interference on the determination of NAR, indicating method specificity.
\end{abstract}

\section{Introduction}

The alternative system of medicine is gaining importance recently. The aim of using plant isolates such as flavonoids, terpenoids, alkaloids, resins, and proanthocyanidins is increasing nowadays. Flavonoids, commonly used in human diet, are a group of naturally occurring polyphenolic compounds that are abundant in many vascular plants. These bioactive compounds have gained interest recently because of their broad pharmacological activities including antioxidant, blood lipid and cholesterol lowering, anti-inflammatory, anticarcinogenic, antiulcer, and antimicrobial, superoxide scavenging actions. Recent attention towards these substances has been stimulated by their potential health benefits [1-7].

Pure herbal constituents are used these days as components in targeted drug delivery system for achieving various pharmaceutical benefits. These flavonoids have prominent bioactivity but the major problems associated with them are limited solubility and permeability accounting for their poor bioavailability [8]. To improve the bioavailability of these components, the use of novel pharmaceutical technology is important. The various novel formulations such as nanosuspensions, liposomes, phytosomes, transfersomes and ethosomes can be prepared for bioactives to demonstrate effective and enhanced drug targeting. The utilization of the novel drug delivery system facilitated the drug administration of phytoconstituents more incisively than conventional drugs. Novel drug delivery system is advantageous due to improvement of bioavailability of the drug by delivering the drug at predetermined rate at the site of action which may reduce the side effects [9].

Naringin (NAR), a citrus flavonoid, is available as one of the most promising compound in human diet. 
Naringin is also proved to enhance the bioavailability of drugs such as paclitaxel and diltiazem when administered concomitantly $[10,11]$. Recently, applications of polyphenols have been of great interest in the area of functional foods, nutraceutical, and pharmaceuticals. Number of chromatographic methods are available for the estimation of naringin in grape and citrus fruits $[12,13]$. There were reports of quantification of naringin in Chinese medicine and Citrus herbs using liquid chromatography [14-16]. Simultaneous diastereomeric separation of naringin and neohesperidin by normal-phase HPLC in commercial samples and herbal medicines was reported [17]. Determination of naringin in orange juice by LC with postcolumn derivatization was reported [18]. There are some LC-MS methods developed for estimation of naringin in citrus juice $[19,20]$. Some of these methods are not validated, some are time-consuming, and some require expensive instruments or laborious extraction techniques and most of the methods used complex mobile phase.

For developing new dosage form of these flavonoids there is need of an analytical methodology to quantify in-process quality control samples, dissolution study samples, and final formulations.

The ambit of the present work was on development of isocratic high performance liquid chromatographic method for estimation of NAR in developed nanoformulations. The developed method was used for quantification of the NAR for assay, content uniformity, solubility studies, and drug release study of the developed formulations. The developed method is simple, precise, accurate, and rapid. The developed method was validated according to the International Conference on Harmonization (ICH) Q2(R1) guideline [21].

\section{Material and Methods}

2.1. Chemicals and Reagents. The working standard, Naringin (99.18\%) was procured from Sigma-Aldrich, USA. Potassium dihydrogen phosphate and orthophosphoric acid AR grade were purchased from Merck India Pvt. Ltd (Mumbai, Maharashtra, India). HPLC solvents like acetonitrile and methanol were procured from Ranchem Ltd, (Mumbai, Maharashtra, India). Ultrapure water was produced in the laboratory from Siemens water purification system. All other chemicals used were of analytical grade.

2.2. Apparatus and Chromatographic Conditions. The analysis was carried out on Shimadzu prominence HPLC separation module with configuration of LC-20AD binary pumps along with DGU-20A5 degasser unit, SPD-20A dual $\lambda \mathrm{UV}$ detector, SPD-M10Avp Photodiode array detector, SIL-20AC HT autosampler, and CTO-10AS vp column oven. System control, data acquisition, and processing were performed with LC Solutions chromatography software (Version 1.24 SP1). Standard substances were weighed on Sartorius CP 225D analytical balance. A glass vacuum-filtration apparatus (Alltech Associates) was employed for the filtration of buffer solution using $0.22 \mu \mathrm{m}$ filter obtained from Pall Pvt. Ltd (Bangalore, India). Degassing of the mobile phase was performed by ultrasonication in Oscar Micro clean-103 Ultrasonic bath.

GraceSmart RP C $18(250.0 \times 4.6 \mathrm{~mm}, 5 \mu \mathrm{m})$ column was used as a stationary phase. The isocratic mobile phase used was acetonitrile and potassium phosphate buffer $(25.0 \mathrm{mM}$; $\mathrm{pH} 3.5 \pm 0.1$, containing $0.2 \%$ triethylamine; $\mathrm{pH}$ adjusted using dilute orthophosphoric acid) in the ratio of $25: 75 \% \mathrm{v} / \mathrm{v}$. The mobile phase was pumped at a flow rate of $1.0 \mathrm{~mL} / \mathrm{min}$. The column was maintained at a temperature of $25^{\circ} \mathrm{C}$ and the eluent was monitored at $282.0 \mathrm{~nm}$. All solutions were injected using acetonitrile: ultrapure water $(1: 1)$ as diluent. The injection volume was $20 \mu \mathrm{L}$ with a total run time of $10.0 \mathrm{~min}$.

\subsection{Method Optimization}

2.3.1. Effect of $p H$. For the present study, separation was carried out on GraceSmart RP $\mathrm{C}_{18}(250.0 \times 4.6 \mathrm{~mm}, 5 \mu \mathrm{m})$ column. The buffer in mobile phase was optimized using $25.0 \mathrm{mM}$ of potassium dihydrogen phosphate containing $0.2 \%$ triethylamine ( $\mathrm{pH}$ adjusted to 3.5 or 7.0 ) and ammonium acetate buffer $(\mathrm{pH} 5.0)$. The analysis of NAR was carried out using $75 \%$ of the buffer in acetonitrile. The chromatographic factors such as capacity factor, theoretical plates, and $10 \%$ asymmetry factor were calculated using chromatographic data software LC Solutions 1.24 SP1.

2.3.2. Effect of Stationary Phases. The standard solution of NAR with concentration of $10.0 \mu \mathrm{g} / \mathrm{mL}$ was injected on various columns such as Water Symmetry $\mathrm{C}_{18}(250.0 \times$ $4.6 \mathrm{~mm}, 5 \mu \mathrm{m})$, Merck Hibar $\mathrm{C}_{18}(250.0 \times 4.6 \mathrm{~mm}, 5 \mu \mathrm{m})$, Kromasil C ${ }_{18}(250.0 \times 4.6 \mathrm{~mm}, 5 \mu \mathrm{m})$, and GraceSmart RP C 18 $(250.0 \times 4.6 \mathrm{~mm}, 5 \mu \mathrm{m})$ and chromatograms were recorded. The chromatographic factors such asymmetry (10\%), capacity factor, and theoretical plates of NAR were monitored.

2.3.3. Effect of Peak Modifier. The standard solution of $10.0 \mu \mathrm{g} / \mathrm{mL}$ of NAR was used for evaluation of the effect of peak modifier in the mobile phase. The chromatogram was recorded with and without peak modifier, that is, $0.2 \%$ triethylamine in phosphate buffer ( $25 \mathrm{mM}$ of buffer adjusted to $\mathrm{pH} 3.5 \pm 0.1$ with dilute orthophosphoric acid). The chromatographic factors such as capacity factor, theoretical plate, and $10 \%$ asymmetry factor were monitored.

2.4. Method Validation. The present method was developed and validated in accordance with the ICH Q2(R1) guideline to fulfil the criteria set by regulatory guidelines

2.4.1. Specificity. To determine the specificity of the developed method in presence of excipients, NAR was spiked with the excipients used for the preparation of nanoformulation. The drug was extracted from these excipients by sonication in methanol for $5 \mathrm{~min}$ followed by filtration through $0.22 \mu \mathrm{m}$ syringe filter. The filtered solution was diluted to obtain solution of $10.0 \mu \mathrm{g} / \mathrm{mL}$ of NAR and injected into the HPLC system. A placebo formulation was processed separately 
as mentioned above and injected into the HPLC system followed by the standard NAR solution $(10.0 \mu \mathrm{g} / \mathrm{mL})$. The developed formulation was also analysed by HPLC-PDA detector to check the peak integrity as peak purity. The chromatograms of placebo formulation and standard NAR were compared to monitor interferences from excipients at the retention time of NAR.

2.4.2. Linearity and Range. The linearity plot was constructed for NAR in the range of 0.1 to $20.0 \mu \mathrm{g} / \mathrm{mL}$. The primary stock solution of $1.0 \mathrm{mg} / \mathrm{mL}$ of NAR was prepared in methanol. From the primary stock solution, secondary stock solution was prepared to get the concentration of $100.0 \mu \mathrm{g} / \mathrm{mL}$ of NAR. Appropriate dilution of the primary and secondary stock solutions was carried in diluent to get concentrations of $0.1,0.2,0.5,1.0,2.0,6.0,10.0$, and $20.0 \mu \mathrm{g} / \mathrm{mL}$ for NAR. The calibration curve was plotted as concentration of the respective drug solutions versus the peak area at each level for five days. The coefficient of determination $\left(r^{2}\right)$, slope, and intercept values were determined and statistically evaluated.

2.4.3. Precision. Precision is the closeness of the analytical results achieved from a set of replicate measurements under the conditions of the method. Precision reflects the random errors which occur in a method [22].

Precision is usually measured as the coefficient of variation or relative standard deviation of analytical results acquired from independently prepared quality control standards.

The precision of the method was determined at three different levels covering entire range of linearity. Three different standards were prepared which contained 0.75, 5.0, and $15.0 \mu \mathrm{g} / \mathrm{mL}$ of NAR.

The intraday precision was evaluated by analysing six sample solutions $(n=6)$ at each level in two different sets in a day. Similarly, the interday precision was evaluated in three consecutive days $(n=18)$. The NAR concentrations were determined and the relative standard deviations (RSD) were calculated.

2.4.4. Accuracy. In order to evaluate the accuracy of the proposed method, a recovery test was performed by adding a known amount of standard solutions to the placebo formulation before extraction, followed by analysis using the proposed method.

The recovery studies were done for three different levels at $80 \%, 100 \%$, and $120 \%$ of assay concentration using standard spiking method.

The placebo formulation was spiked with 8.0, 10.0, and $12.0 \mu \mathrm{g} / \mathrm{mL}$ of standard NAR. The prepared samples were analysed using proposed chromatographic conditions. The amount recovered was using the linearity curve.

The percentage of recovery was calculated according to the following formula:

$$
\operatorname{Recovery}(\%)=\frac{(\text { Amount found in spiked placebo sample })}{\text { spiked amount }} \times 100
$$

2.4.5. Limit of Detection (LOD) and Limit of Quantitation (LOQ). LOD is the ability of analytical method to detect the lowest concentration of the analyte. LOQ is lowest concentration of the analyte which can be quantitatively determined with acceptable precision and accuracy.

The LOD and LOQ were determined by the following equation according to ICH guideline:

$$
\begin{aligned}
& \text { LOD }=3.3 \times \sigma / s, \\
& \text { LOQ }=10 \times \sigma / s, \\
& \sigma-\text { Standard deviation of blank response, } \\
& s-\text { Slope of regression equation. }
\end{aligned}
$$

2.4.6. Robustness. The importance of establishing robustness of the developed method by the comparison of series of system suitability parameters obtained by employing deliberate changes is to ensure the integrity of analytical procedure whenever it is used. In the present study, the assay concentration $10.0 \mu \mathrm{g} / \mathrm{mL}$ of NAR was used for the determination of the robustness of the method. The following parameters were considered for the robustness:

(i) effect of $\mathrm{pH}$ of buffer in mobile phase $( \pm 0.2)$,

(ii) effect of mobile phase composition $( \pm 2 \%)$, (iii) effect of wave length $( \pm 2 \mathrm{~nm})$,

(iv) effect of flow rate $( \pm 10 \%)$.

2.4.7. Solution Stability. NAR belongs to the category of flavonoids which calls for checking its stability. It is necessary to carry out the stability of bioactive compounds given their property of undergoing various forms of degradation. The solution stability of NAR was carried out at bench top, refrigerated condition and in autosampler. Stock solution of $100.0 \mu \mathrm{g} / \mathrm{mL}$ of NAR was prepared in methanol. The solution was diluted using diluent to get the concentration of $10.0 \mu \mathrm{g} / \mathrm{mL}$ of NAR and subjected to bench top and autosampler stability conditions. The stock solution was subjected to refrigerated stability condition and monitored for 15 days as long-term stability. The samples after being subjecting to various stability conditions were injected into the HPLC system. The concentration was determined at predetermined time interval using freshly prepared calibration curve.

2.4.8. System Suitability. The purpose of the system suitability test is to ensure that the complete testing system (including instrument, reagents, columns, and analysts) is suitable for the intended application. 
TABLE 1: System suitability parameters.

\begin{tabular}{lcc}
\hline Parameter & Limit & Observed value \\
\hline Capacity factor & $k^{\prime}>2$ & $1.804 \pm 0.002$ \\
Injection precision & $\mathrm{RSD}<1 \%$ for $n>6$ & 0.264 \\
Tailing factor & $T<2$ & $1.04 \pm 0.02$ \\
Theoretical plates/meter & $N>2000$ & $9646.5 \pm 154.3$ \\
\hline
\end{tabular}

System suitability testing is an integral part of liquid chromatographic methods which is used to verify the reproducibility of the chromatographic system for the analysis to be done. The tests are based on the concept that the equipment, electronics, analytical operations, and samples to be analysed constitute an integral system that can be evaluated as such.

In the current US-FDA guidelines on "Validation of chromatographic methods," the following acceptance limits are proposed as initial criteria (Table 1). For the present study, $10.0 \mu \mathrm{g} / \mathrm{mL}$ of NAR was injected six times to record the system suitability parameters.

\section{Application of Method}

Developed method was applied for quantification of the NAR in developed nanosuspension prepared using Lutrol F68 (stabilizer) by cavi-precipitation method. Various formulations were prepared by altering drug and stabilizer ratio. The nanosuspensions were lyophilized using mannitol as cryoprotectant and used further for characterization. $10 \mathrm{mg}$ equivalent quantity of lyophilized formulation was weighed and transferred to $10 \mathrm{~mL}$ volumetric flask. $3 \mathrm{~mL}$ of methanol was added to the flask and sonicated for $10 \mathrm{~min}$. The volume was made up to the mark by using methanol. The solution was filtered through $0.22 \mu \mathrm{m}$ syringe filter. The filtrate was further diluted to get the concentration equivalent to $10.0 \mu \mathrm{g} / \mathrm{mL}$ in diluent. The resulting solution was analysed by proposed HPLC method.

\section{Results and Discussion}

The objective of the present study was to develop and validate liquid chromatographic method for estimation of NAR in the developed formulations.

The proposed HPLC method is simple with less time consumption and requires fewer reagents. This method could be used in quality control test and estimation of the release study samples in pharmaceutical industries. The retention time of NAR was found to be $7.46 \pm 0.5 \mathrm{~min}$ (Figure 1).

The developed method was validated as per the $\mathrm{ICH}$ Q2(R1) guideline to check the reliability of the method.

4.1. Effect of $p H$. By attempting the various $\mathrm{pH}$ of the mobile phase ( $\mathrm{pH} 3.5,5.0$, and 7.0), it was observed that the retention time for NAR was found to be around $7.4 \mathrm{~min}$ in all the above mentioned $\mathrm{pH}$ and there was no significant effect of $\mathrm{pH}$ on the retention time. The peak shape was found to be good in $\mathrm{pH}$ 3.5 compared to $\mathrm{pH} 5.0$ and 7.0. Hence, for the present study $\mathrm{pH} 3.5$ was selected having the advantage of offering very

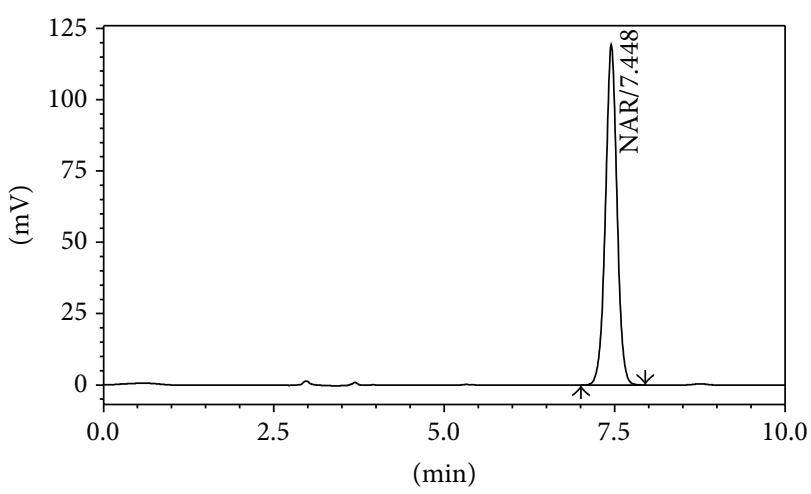

FIGURE 1: Representative chromatogram of standard NAR.

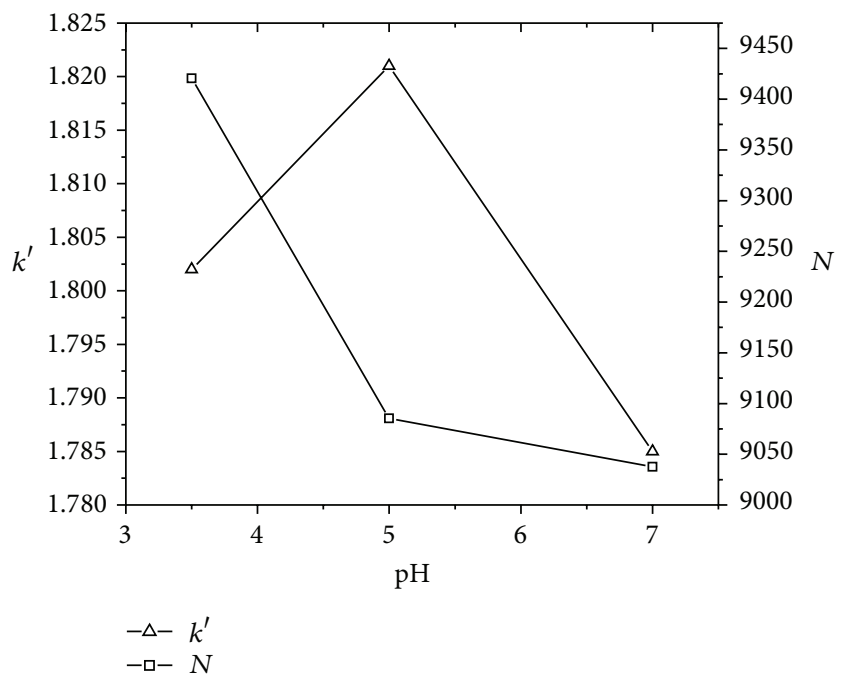

FIGURE 2: Effect of $\mathrm{pH}$ of capacity factor and theoretical plate of standard NAR.

good buffering capacity and maintaining NAR in unionized state. The effect of $\mathrm{pH}$ on capacity factor and theoretical plates of NAR is shown in Figure 2. The capacity factor was found to be stationed around 1.8 and with highest theoretical plate count at pH 3.5 with desired run time. The chromatographic parameters of NAR standard at various $\mathrm{pH}$ were tabulated in Table 4.

4.2. Effect of Stationary Phases. The retention time of NAR on waters Symmetry $\mathrm{C}_{18}(250 \times 4.6 \mathrm{~mm}, 5 \mu \mathrm{m})$ column was found to be less compared to Merck Hibar $\mathrm{C}_{18}(250 \times 4.6 \mathrm{~mm}, 5 \mu \mathrm{m})$, Kromasil $\mathrm{C}_{18}(250 \times 4.6 \mathrm{~mm}, 5 \mu \mathrm{m})$, and GraceSmart RP $\mathrm{C}_{18}$ $(250 \times 4.6 \mathrm{~mm}, 5 \mu \mathrm{m})$ columns. The peak was broader and the column efficiency was less in case of Kromasil and Merck Hibar column leaving them unconsidered for the present study.

There was no significant change in the retention time of the NAR in all the above columns. The GraceSmart $\mathrm{RP} \mathrm{C}_{18}(250 \times 4.6 \mathrm{~mm}, 5 \mu \mathrm{m})$ column showed good peak shape, less tailing, and acceptable retention time with better column efficiency compared to all other columns. Hence, in 
the present study GraceSmart RP $\mathrm{C}_{18}(250.0 \times 4.6 \mathrm{~mm}, 5 \mu \mathrm{m})$ was selected as the column for the analysis. The effect of column on chromatographic factors mainly capacity factor and theoretical plates was represented in Figure 3. The highest capacity factor that is 1.8 with highest theoretical plates count was observed with GraceSmart RP $\mathrm{C}_{18}(250.0 \times 4.6 \mathrm{~mm}$, $5 \mu \mathrm{m})$. The results for system suitability parameters were tabulated in Table 4.

4.3. Effect of Peak Modifier. It was observed that, the $10 \%$ asymmetry factor was found to be 1.287 without TEA with low theoretical plate count. In presence of TEA the tailing factor $(10 \%)$ was 1.045 with better column efficiency. The improvement of column efficiency with peak shape may be due to capping of the acidic functional groups of stationary phase resulting in the reduction of interaction of hydroxyl functional group of NAR releasing the drug quicker. Considering the optimal results, $0.2 \%$ triethylamine was used as peak modifier in the mobile phase. Results of chromatographic parameters were tabulated in Table 4.

\subsection{Validation of the Method}

4.4.1. Specificity. The present method was found to be highly specific as there was no interference at retention time of the NAR from the placebo formulation.

The specificity of the method was investigated by conducting a photodiode-array analysis to investigate the integrity of the drug peaks and to clarify the purity of the peaks. The total peak purity was monitored for NAR. The peak purity was found to be greater than the peak purity threshold which indicated that the peak of NAR was pure and there was no interference from excipients. In the chromatograms of the formulations, some additional peaks were observed which may be due to presence of excipients in the formulations. The chromatogram, spectrum with peak purity chart is shown in Figure 4.

4.4.2. Linearity. The response for the detector was determined to be linear over the range of 0.1 to $20.0 \mu \mathrm{g} / \mathrm{mL}$ for NAR. The calibration curve was plotted as concentration of the drug versus the response (area of the drug peak) at each level. The proposed method was evaluated by its coefficient of determination and intercept value calculated in the statistical study. They were represented by the linear regression equation as $y=27494 x+32335$ and " $r$ " value $=$ 0.99995. For all the calibration curves the coefficient of determination was within the limit of $0.9999585 \leq r^{2} \geq$ 0.9999960 .

Slopes and intercepts were obtained by using regression equation $(y=m x+c)$ and least square treatment of the results were used to confirm linearity of the developed method.

4.4.3. Precision. The $\% \mathrm{CV}$ of interday and intraday precision obtained was less than $1 \%$ for NAR. The precision was carried out at three different concentrations of NAR over the linearity. The intraday and interday precision of NAR was in the range of $0.188-0.291$ and $0.264-0.891$, respectively (Table 2 ). From

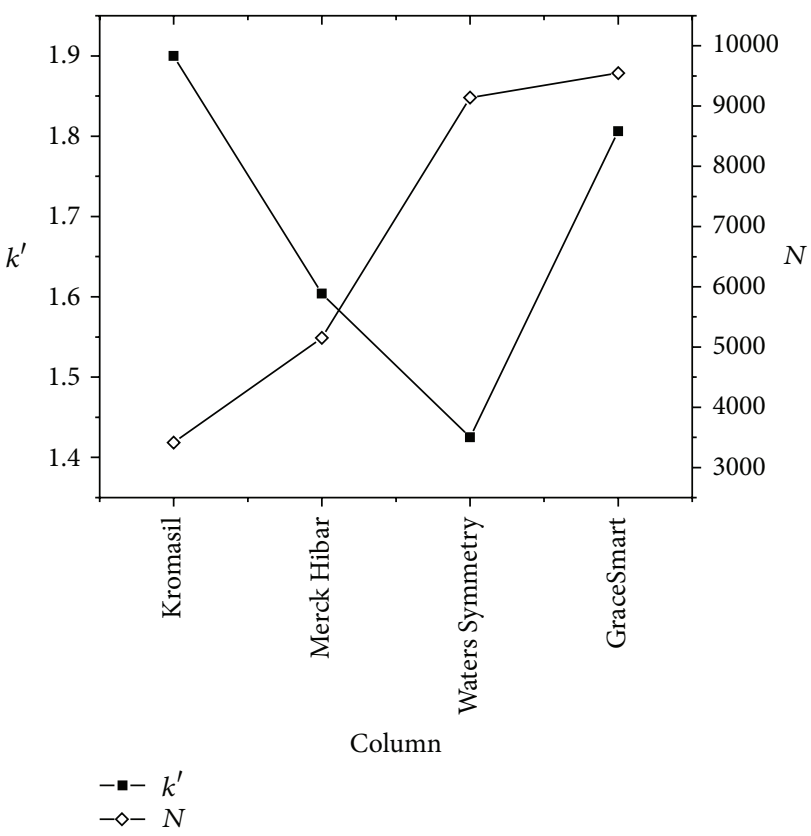

FIGURE 3: Effect of column on capacity factor and theoretical plate on standard NAR.

TABLE 2: The precision data of NAR by the proposed HPLC method.

\begin{tabular}{lcc}
\hline \multirow{2}{*}{ Concentration $(\mu \mathrm{g} / \mathrm{mL})$} & \multicolumn{2}{c}{ \% RSD } \\
& Intraday $(n=12)$ & Interday $(n=18)$ \\
\hline 0.75 & 0.291 & 0.891 \\
5.0 & 0.188 & 0.784 \\
15.0 & 0.278 & 0.264 \\
\hline
\end{tabular}

TABLE 3: The accuracy data of NAR by the proposed HPLC method.

\begin{tabular}{lcccc}
\hline Spiked level & $\begin{array}{c}\text { Amount } \\
\text { added }\end{array}$ & $\begin{array}{c}\text { Amount } \\
\text { recovered }\end{array}$ & \% recovery & $\begin{array}{c}\text { Mean \% } \\
\text { recovery }\end{array}$ \\
\hline \multirow{3}{*}{$80 \%$} & 7.86 & 7.79 & 99.11 & \\
& 7.83 & 7.76 & 99.11 & $99.19 \pm 0.15$ \\
& 7.83 & 7.78 & 99.36 & \\
$100 \%$ & 10.05 & 10.01 & 99.60 & \\
& 10.05 & 9.99 & 99.40 & $99.50 \pm 0.10$ \\
& 10.06 & 10.01 & 99.50 & \\
\hline \multirow{4}{*}{$120 \%$} & 12.04 & 12.07 & 100.25 & \\
& 12.000 & 11.817 & 98.48 & $99.30 \pm 0.89$ \\
& 11.97 & 11.87 & 99.16 & \\
\hline \multirow{5}{*}{$\%$ mean recovery } & & $99.33 \pm 0.16$ \\
\hline
\end{tabular}

the data obtained, the developed HPLC method was found to be highly precise.

4.4.4. Accuracy. The assay concentration for NAR was considered to be $10.0 \mu \mathrm{g} / \mathrm{mL}$. The recovery was calculated using placebo spiking method at three levels of $80-120 \%$ of 


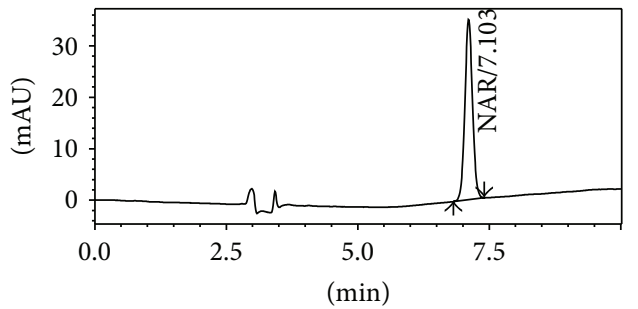

(a)

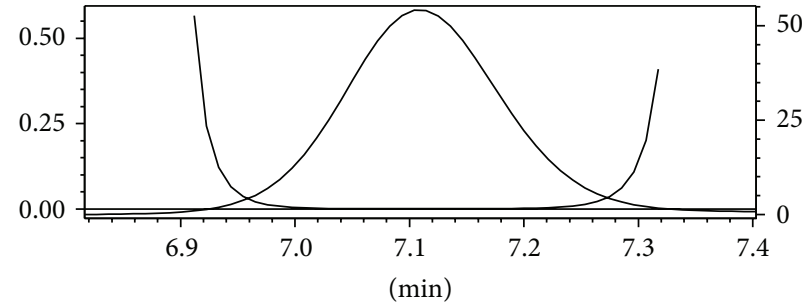

(b)

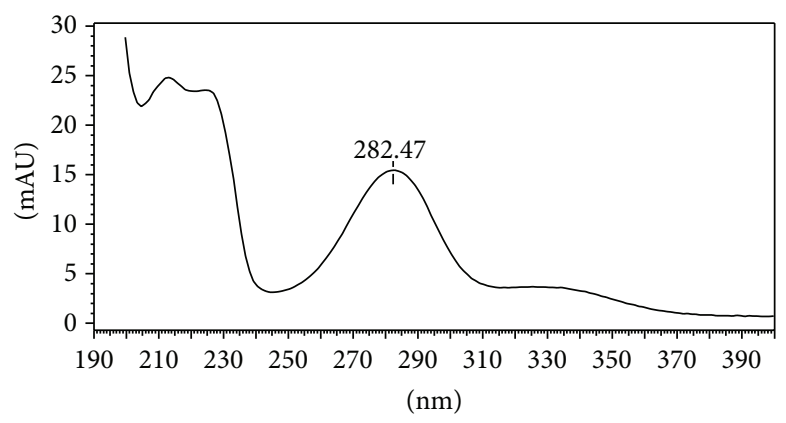

(c)

FIGURE 4: Representative chromatogram of naringin nanoformulation sample (a), and peak purity graph (b), and PDA spectrum (c).

TABLE 4: System suitability parameter.

\begin{tabular}{lccccccccc}
\hline \multirow{2}{*}{ Parameters } & \multicolumn{3}{c}{ Mobile phase $\mathrm{pH}$} & \multicolumn{3}{c}{ Column } \\
& 3.5 & 5.0 & 7.0 & Kromasil & Merck Hibar & Waters Symmetry & GraceSmart & Without TEA & With 0.2\% v/v TEA \\
\hline$R_{t}$ & 7.467 & 7.493 & 7.396 & 7.702 & 6.917 & 6.442 & 7.452 & 7.443 & 7.484 \\
$T$ & 1.054 & 1.048 & 1.052 & 0.936 & 1.242 & 1.127 & 1.0365 & 1.287 & 1.045 \\
$k^{\prime}$ & 1.802 & 1.821 & 1.785 & 1.900 & 1.604 & 1.425 & 1.806 & 1.804 & 1.803 \\
$N$ & 9420.4 & 9085.4 & 9037.6 & 3415.11 & 5152.9 & 9139.41 & 9546.5 & 7687.4 & 9434.5 \\
\hline
\end{tabular}

$R_{t}$ : Peak retention time, $T$ : tailing factor, $k^{\prime}$ : capacity factor and $N$ : theoretical plates.

TABLE 5: Robustness parameter of the proposed method.

\begin{tabular}{lc}
\hline Parameters & \% RSD of area of NAR \\
\hline Mobile phase $\mathrm{pH}$ & \\
3.3 & 0.113 \\
3.7 & 0.287 \\
Mobile phase composition & \\
(acetonitrile : buffer) $(\% \mathrm{v} / \mathrm{v})$ & \\
$27: 73$ & 0.252 \\
$23: 77$ & 0.166 \\
UV detector wavelength $(\mathrm{nm})$ & \\
280 & 0.107 \\
284 & 0.102 \\
Flow rate $(\mathrm{mL} / \mathrm{min})$ & \\
0.9 & 0.097 \\
1.1 & 0.363 \\
\hline
\end{tabular}

the assay concentration. The mean recovery for the NAR was $99.33 \pm 0.16 \%$. The recovery data for the NAR is shown in Table 3.

4.4.5. Quantification and Detection Limit. The LOD and LOQ of NAR were found to be $17.0 \mathrm{ng} / \mathrm{mL}$ and $50.0 \mathrm{ng} / \mathrm{mL}$, respectively.

4.4.6. Robustness. Method robustness was checked for NAR estimation after employing the variables to the optimised method. The data generated from the proposed method had significantly demonstrated that the HPLC method developed is robust. Results of the robustness parameter were tabulated in Table 5. It was observed that the $\%$ RSD for all the variables parameters was $<1 \%$, indicating the method is robust.

4.4.7. Solution Stability. While determining the solution stability of NAR, it was observed that the methanolic solution of 
NAR is stable at bench top and autosampler for $12 \mathrm{~h}$ and $24 \mathrm{~h}$, respectively. Also NAR was stable at refrigerated condition for 15 days.

\section{Application of the Method}

The proposed method was utilized for the estimation of NAR in developed formulations. The nanoformulation assay was found in the range of $60-98 \%$ for different batches. The present method was also utilized for in vitro release of the developed formulations indicating the suitability of the method.

\section{Conclusion}

The proposed HPLC method for estimation of the naringin in developed nanoformulations was applied successfully. Specificity oriented method development has given a wide edge to this method for the estimation of naringin in many other formulations. Utilizing an isocratic mobile phase with commonly used column is very easy to perform to give acceptable and reproducible results. The validation of proposed method was carried out as per ICH guideline and performance data for all the parameters tested is acceptable. LOD and LOQ, established by this method, are lesser than previously described methods. The method is found to be linear in the specified range, precise, and robust. Accuracy of the method is also established for the formulation. Hence, the proposed method is rapid, simple, and can be applied to quality control analyses of formulated product.

\section{Conflict of Interests}

The authors declare that there is no conflict of interests regarding the publication of this paper.

\section{Acknowledgment}

The authors are grateful to Manipal University for providing the financial support under Dr. T. M. Pai Endowment Chair fund for carrying out this research work.

\section{References}

[1] F. I. Kanaze, E. Kokkalou, M. Georgarakis, and I. Niopas, "A validated solid-phase extraction HPLC method for the simultaneous determination of the citrus flavanone aglycones hesperetin and naringenin in urine," Journal of Pharmaceutical and Biomedical Analysis, vol. 36, no. 1, pp. 175-181, 2004.

[2] H.-J. Kim, G. T. Oh, Y. B. Park, M.-K. Lee, H.-J. Seo, and M.-S. Choi, "Naringin alters the cholesterol biosynthesis and antioxidant enzyme activities in LDL receptor-knockout mice under cholesterol fed condition," Life Sciences, vol. 74, no. 13, pp. 1621-1634, 2004.

[3] M. J. Martin, E. Marhuenda, C. Perez-Guerrero, and J. M. Franco, "Antiulcer effect of naringin on gastric lesions induced by ethanol in rats," Pharmacology, vol. 49, no. 3, pp. 144-150, 1994.
[4] S.-M. Jeon, S.-H. Bok, M.-K. Jang et al., "Antioxidative activity of naringin and lovastatin in high cholesterol-fed rabbits," Life Sciences, vol. 69, no. 24, pp. 2855-2866, 2001.

[5] Y. T. Chen, R. L. Zheng, Z. J. Jia, and Y. Ju, "Flavonoids as superoxide scavengers and antioxidants," Free Radical Biology and Medicine, vol. 9, no. 1, pp. 19-21, 1990.

[6] T. P. T. Cushnie and A. J. Lamb, "Antimicrobial activity of flavonoids," International Journal of Antimicrobial Agents, vol. 26, no. 5, pp. 343-356, 2005.

[7] L. H. Yao, Y. M. Jiang, J. Shi et al., "Flavonoids in food and their health benefits," Plant Foods for Human Nutrition, vol. 59, no. 3, pp. 113-122, 2004.

[8] K. Kesarwani and R. Gupta, "Bioavailability enhancers of herbal origin: an overview," Asian Pacific Journal of Tropical Biomedicine, vol. 3, no. 4, pp. 253-266, 2013.

[9] K. Gowthamarajan, L. P. Dwarampudi, S. Ramaswamy, and B. Suresh, "The recent surge in herbal pharmaceuticals," PharmaTimes, vol. 45, no. 9, pp. 25-28, 2013.

[10] J.-S. Choi and S.-C. Shin, "Enhanced paclitaxel bioavailability after oral coadministration of paclitaxel prodrug with naringin to rats," International Journal of Pharmaceutics, vol. 292, no. 1-2, pp. 149-156, 2005.

[11] J.-S. Choi and H.-K. Han, "Enhanced oral exposure of diltiazem by the concomitant use of naringin in rats," International Journal of Pharmaceutics, vol. 305, no. 1-2, pp. 122-128, 2005.

[12] I. A. Ribeiro and M. H. L. Ribeiro, "Naringin and naringenin determination and control in grapefruit juice by a validated HPLC method," Food Control, vol. 19, no. 4, pp. 432-438, 2008.

[13] S. Gorinstein, D. Huang, H. Leontowicz et al., "Determination of naringin and hesperidin in citrus fruit by high-performance liquid chromatography. The antioxidant potential of citrus fruit," Acta Chromatographica, no. 17, pp. 108-124, 2006.

[14] F. I. Kanaze, C. Gabrieli, E. Kokkalou, M. Georgarakis, and I. Niopas, "Simultaneous reversed-phase high-performance liquid chromatographic method for the determination of diosmin, hesperidin and naringin in different citrus fruit juices and pharmaceutical formulations," Journal of Pharmaceutical and Biomedical Analysis, vol. 33, no. 2, pp. 243-249, 2003.

[15] H. Zhang, S. Chen, F. Qin, X. Huang, P. Ren, and X. Gu, "Simultaneous determination of 12 chemical constituents in the traditional Chinese Medicinal Prescription Xiao-Yao-San-JiaWei by HPLC coupled with photodiode array detection," Journal of Pharmaceutical and Biomedical Analysis, vol. 48, no. 5, pp. 1462-1466, 2008.

[16] P. Mouly, E. M. Gaydou, and A. Auffray, "Simultaneous separation of flavanone glycosides and polymethoxylated flavones in citrus juices using liquid chromatography," Journal of Chromatography A, vol. 800, no. 2, pp. 171-179, 1998.

[17] N. Uchiyama, I. H. Kim, R. Kikura-Hanajiri, N. Kawahara, T. Konishi, and Y. Goda, "HPLC separation of naringin, neohesperidin and their C-2 epimers in commercial samples and herbal medicines," Journal of Pharmaceutical and Biomedical Analysis, vol. 46, no. 5, pp. 864-869, 2008.

[18] A. Andreu-Navarro, J. M. Fernández-Romero, and A. GómezHens, "Luminescent determination of flavonoids in orange juices by LC with post-column derivatization with aluminum and terbium," Journal of Separation Science, vol. 33, no. 4-5, pp. 509-515, 2010.

[19] L. Ding, X. Luo, F. Tang, J. Yuan, Q. Liu, and S. Yao, "Simultaneous determination of flavonoid and alkaloid compounds 
in Citrus herbs by high-performance liquid chromatographyphotodiode array detection-electrospray mass spectrometry," Journal of Chromatography B, vol. 857, no. 2, pp. 202-209, 2007.

[20] Z. Aturki, V. Brandi, and M. Sinibaldi, "Separation of flavanone7-O-glycoside diastereomers and analysis in citrus juices by multidimensional liquid chromatography coupled with mass spectrometry," Journal of Agricultural and Food Chemistry, vol. 52, no. 17, pp. 5303-5308, 2004.

[21] International Conference on the Harmonization of Technical Requirements for the Registration of Pharmaceuticals for Human Use. ICH Harmonized Tripartite Guideline Validation of Analytical Procedures: Text and Methodology Q2 (R1), Current Step 4 version, Parent Guideline dated 27 October 1994 (Complementary Guideline on Methodology dated 6 November 1996 incorporated in November 2005), 2005.

[22] Guidance for the Validation of Analytical Methodology and Calibration of Equipment Used for Testing of Illicit Drugs in Seized Materials and Biological Specimen, Laboratory and Scientific Section United Nations Office on Drugs and Crime Vienna, United Nations, New York, NY, USA, 2009. 

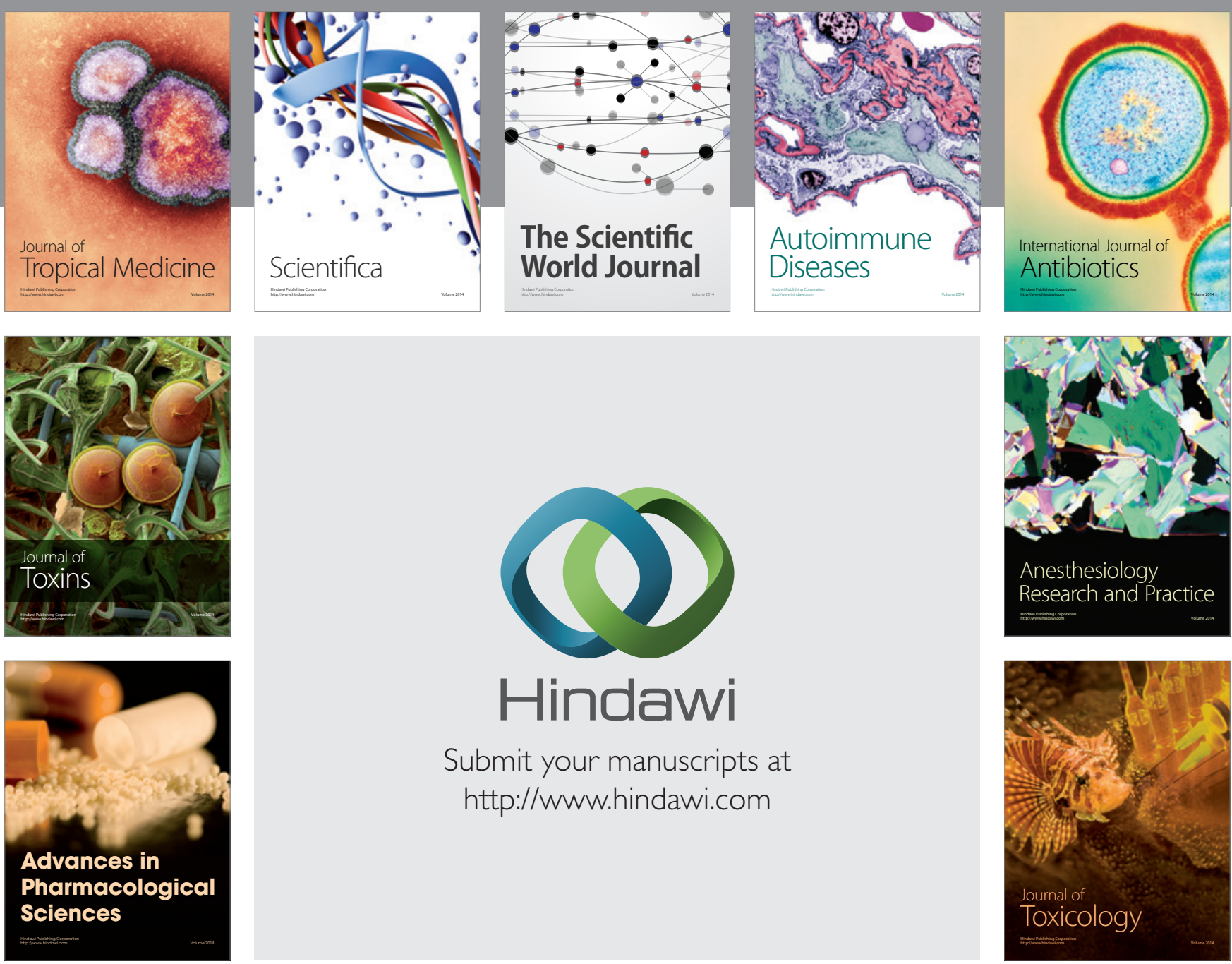

\section{Hindawi}

Submit your manuscripts at

http://www.hindawi.com
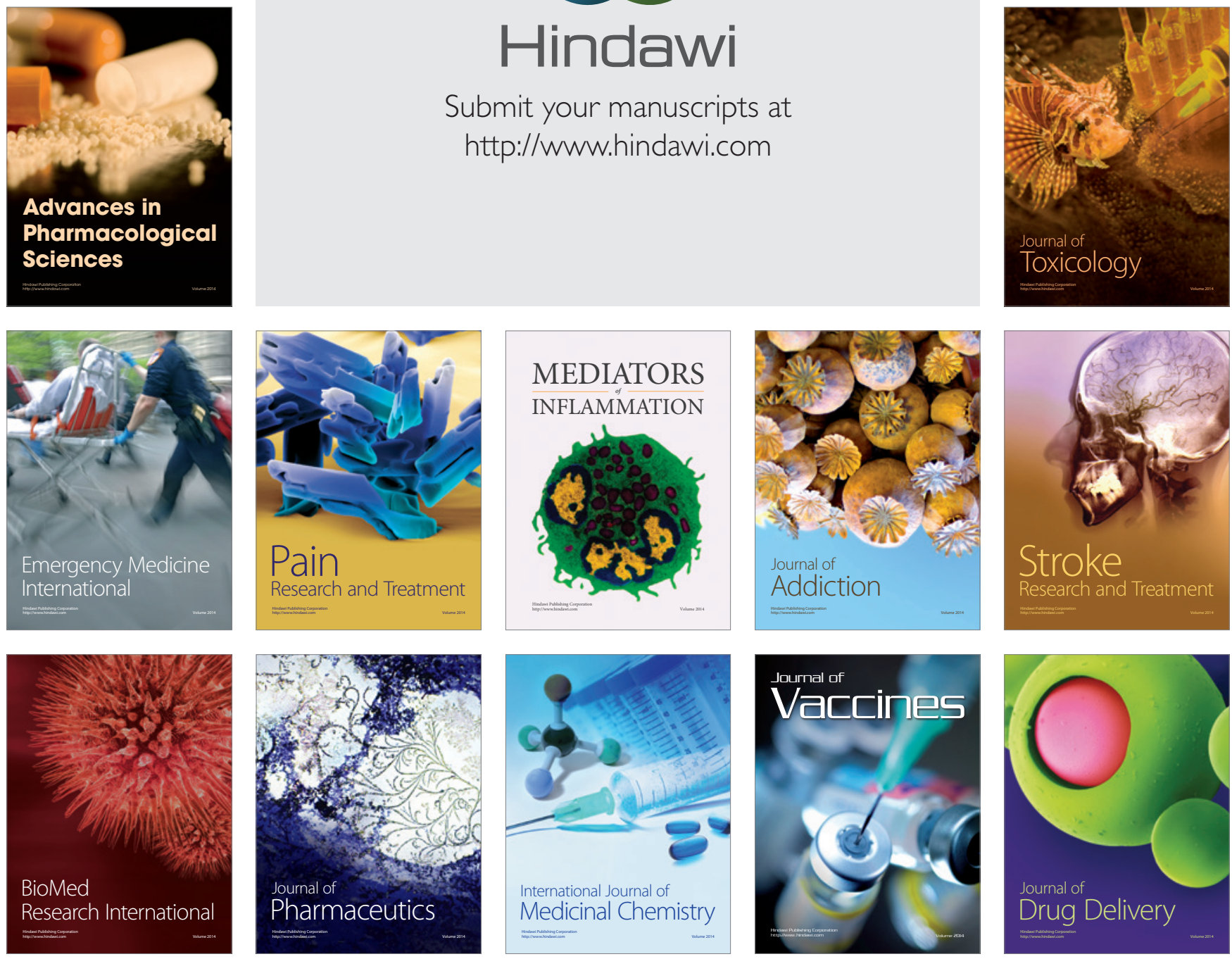